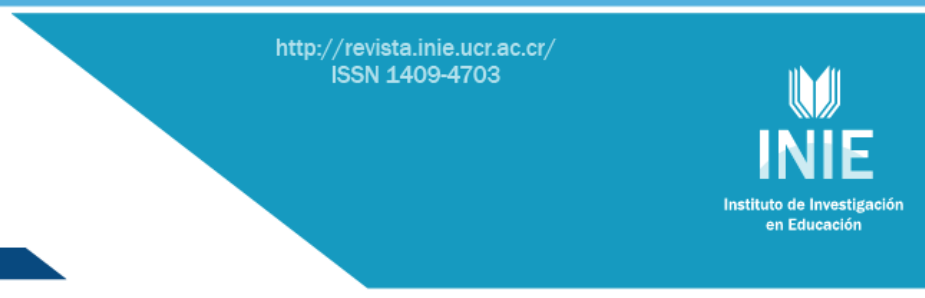

\title{
DISEÑO Y VALIDACIÓN DE UNA ESCALA PARA EVALUAR LAS ESTRATEGIAS DE GESTIÓN E INTERVENCIÓN DOCENTE EN EDUCACIÓN PRIMARIA
}

DESIGN AND VALIDATION OF A SCALE WITH WHICH TO EVALUATE THE MANAGEMENT STRATEGIES AND EDUCATIONAL INTERVENTION IN PRIMARY EDUCATION

\section{Volumen 15, Número 3}

Setiembre - Diciembre

pp.1-24

Este número se publicó el $1^{\circ}$ de setiembre de 2015

DOI: http://dx.doi.org/10.15517/aie.v15i3.20927

Inmaculada Chiva Sanchis

Genoveva Ramos Santana

Revista indizada en REDALYC, $\underline{\text { SCIELO }}$

Revista distribuida en las bases de datos:

CATÁLOGO DE LATINDEX, IRESIE, CLASE, DIALNET, DOAJ, E-REVIST@S, SHERPA/ROMEO, QUALIS, MIAR

Revista registrada en los directorios:

ULRICH'S, REDIE, RINACE, OEI, MAESTROTECA, PREAL, $\underline{\text { CLACSO }}$ 


\title{
DISEÑO Y VALIDACIÓN DE UNA ESCALA PARA EVALUAR LAS ESTRATEGIAS DE GESTIÓN E INTERVENCIÓN DOCENTE EN EDUCACIÓN PRIMARIA

\author{
DESIGN AND VALIDATION OF A SCALE WITH WHICH TO EVALUATE THE \\ EDUCATION
} MANAGEMENT STRATEGIES AND EDUCATIONAL INTERVENTION IN PRIMARY
}

\author{
Inmaculada Chiva Sanchis ${ }^{1}$ \\ Genoveva Ramos Santana ${ }^{2}$
}

\begin{abstract}
Resumen: Este artículo aporta evidencias de validación de una escala de valoración destinada a evaluar las estrategias de gestión e intervención docente, también llamadas metodologías docentes, empleadas por el profesorado de primaria. Esta investigación es realizada desde dos aproximaciones: una cuantitativa basada en un estudio de encuesta, donde se recogen las valoraciones de estudiantes acerca de cómo se aprende en el aula y qué recursos, actividades y sistemas de evaluación utiliza el profesorado; y una cualitativa basada en las aportaciones de un comité de personas expertas. Los análisis realizados atienden a la validez de contenido y de constructo de la escala así como a la fiabilidad de sus ítems. La validación de dicha escala se realizó con un total de 9 centros de la Comunidad Valenciana (España), en el nivel de 6o de primaria, concretamente 324 estudiantes. Los resultados permiten, por un lado, comprobar el alto nivel de consistencia interna de la escala de valoración y, por otro lado, presentar un instrumento destinado a la autoevaluación y/o heteroevaluación que ofrezca al profesorado la información necesaria para modificar y mejorar su gestión e intervención en el aula para adaptarse mejor a las competencias y necesidades de sus estudiantes en un contexto de formación permanente.
\end{abstract}

Palabras clave: METODOLOGÍA DOCENTE, EDUCACIÓN PRIMARIA, VALIDEZ, ESCALAS, ESPAÑA

\begin{abstract}
This article provides evidence of validation of a rating scale intended to evaluate management strategies and educational intervention, also called teaching methodologies, used by primary teachers. This research is conducted from two approaches: a quantitative study based on a survey, where valuations students about how they learn in the classroom and what resources, activities and evaluation systems are collected using teachers; and a qualitative based on input from a committee of experts. The analyzes serve content validity and construct the scale and the reliability of its items. The validation of the scale was conducted with a total of 9 centers of Valencia (Spain), in the 6th grade level, namely 324 students. The results allow, on the one hand, to check the high level of internal consistency of the scale of assessment, and on the other hand, present a tool for self-assessment and / or hetero offering teachers the information needed to modify and improve their management and intervention in the classroom for better match the skills and needs of their students in a context of lifelong learning.
\end{abstract}

Key words: TEACHING METHODOLOGY, PRIMARY EDUCATION, VALIDITY, SCALE, SPAIN

\footnotetext{
1 Profesora Universitaria del Departamento MIDE (Métodos de Investigación y Diagnóstico en Educación), Facultad de Filosofía y Ciencias de la Educación, Universitat de València, España. Dirección electrónica: inmaculada.chiva@uv.es

2 Profesora Universitaria en el Departamento MIDE en la Facultad de Filosofía y Ciencias de la Educación de la Universitat de València, España.Dirección electrónica: Genoveva.ramos@uv.es
}

Artículo recibido: 8 de noviembre, 2014

Enviado a corrección: 7 de mayo, 2015

Aprobado: 17 de agosto, 2015 


\section{Introducción}

Las estrategias de gestión e intervención docente que el profesorado implementa en las aulas son un elemento de preocupación constante para los investigadores e investigadoras educativas $y$, por supuesto, para el propio profesorado (Mendoza y Covarrubias, 2014). Entre los motivos que llevan a esta preocupación se encuentra el hecho de ser una variable que influye en el rendimiento o logros académicos del alumnado (Jornet y Backhoff, 2004; Tourón, 2000; Arribas, 2012) y en el desarrollo de sus competencias básicas. El actual modelo educativo exige prácticas docentes que se centren en la planificación, diseño e implementación de metodologías de enseñanza- aprendizaje activas para alcanzar el desarrollo integral de las competencias de los alumnos y alumnas (Lira, 2010).

Es por ello que las estrategias de gestión e intervención docente o las metodologías docentes han pasado a ser uno de los focos de atención más estudiados en los últimos años en cualquier nivel educativo (Carrera y Marín, 201; De Miguel, 2006; Garrote, 2008; Pozo, 2008) desde un enfoque más interdisciplinar, pues las competencias que los estudiantes han de desarrollar se consiguen con todas las áreas de conocimiento aunque algunas tengan vinculaciones muy evidentes con campos determinados (Crespo, 2011). Sin embargo, aunque es un tema relevante y actual, esto no está sucediendo de igual forma en el nivel de educación primaria, pues como señalan distintos investigadores en esta etapa los estudios se centran sobre todo en innovaciones metodológicas concretas y circunscritas a una materia (Bermejo, 2006; Murillo, 2007; Paredes Labra, 1999; Robles, Alfageme y Vallejo, 2011).

Creemos que la metodología de enseñanza-aprendizaje es responsabilidad de los centros educativos y del profesorado aunque las actuales normativas educativas incorporan unos principios metodológicos de carácter general para cada etapa. En educación primaria, la organización en áreas se ha de entender sin perjuicio del carácter global de la etapa, dada la necesidad de integrar las distintas experiencias y aprendizajes del alumnado de estas edades, de ahí que la acción educativa deba integrar dichas experiencias y aprendizajes adaptándose al ritmo de trabajo de los y las estudiantes (MEC, 2006).

Al igual que autores como Ademar y Ariel (2008) somos conscientes de que la educación primaria ha ido adquiriendo, a través del tiempo, un carácter específico y particular, ya no se puede considerar al alumnado de primaria como una persona que recibe pasivamente las explicaciones de los maestros y maestras. Hoy, el alumnado de primaria se 
ha transformado en personas activas, inquietas, capaces de argumentar, de discutir, de dar razones, de exigir atención, sensibilizadas por los acontecimientos que les rodean y por los distintos modos de acceder a los conocimientos y a la información. Alumnado con el que se trabaja el desarrollo de un conjunto de competencias desde las asignaturas, áreas o espacios curriculares a través de distintas metodologías de aprendizaje-enseñanza (Ademar y Ariel, 2008; Segura, 2009). Metodologías que han de trabajar desde la socialización, globalización, participación, creatividad, autonomía y responsabilidad desde una pedagogía activa, con el trabajo en equipo, el aprendizaje cooperativo, las tecnologías de la información y la comunicación, entre otros elementos.

Ante esta situación, creemos que es pedagógico preguntarse si las metodologías docentes o de las estrategias de gestión e intervención que se utilizan en el aula pueden valorarse a través de un instrumento o escala, como estrategia de diagnóstico o evaluación acerca de cómo se trabaja en el aula y, a partir de sus resultados, descubrir qué elementos deben ser modificados para favorecer la formación de las competencias del alumnado y por ende sus logros académicos. "El docente competente es aquel que sabe observar todo aquello que interesa al alumnado para conjugarlo en favor de su formación" (Crespo, 2011, p. 53), pero añade a sus competencias, docentes, la capacidad de trabajo y aprendizaje continuo mejorando su quehacer diario. Estas cuestiones hacen que hayamos visto la metodología docente como una variable diferencial que puede llevarnos a identificar importantes elementos de mejora por su influencia en el rendimiento y en el desarrollo de las competencias de los alumnos y de las alumnas de educación primaria.

Desde este contexto, y con estos intereses descritos, se desencadenan dos proyectos de investigación I+D (Innovación y Desarrollado), denominados AVACO (Análisis de Variables de Contexto: diseño de cuestionarios de contexto para la evaluación de sistemas educativos) y MAVACO (Modelo de Análisis de Variables de Contexto para la evaluación de sistemas educativos), implementados desde el año 2007 hasta el 2014 por el Grupo GEM (Grupo de Evaluación y Medición) de la Universitat de València (España). Estos proyectos se centraron en la revisión de variables diferenciales a atender en los planes de evaluación de sistemas educativos.

De estas variables, la metodología docente utilizada en el aula aparecía como una de las más influyentes y, por tanto, con una gran capacidad para explicar el rendimiento académico del estudiante. Por todo ello, nos planteamos, a partir de nuestros conocimientos en el área específica de la evaluación educativa, la necesidad de diseñar y validar una 
escala de valoración dirigida a recoger la percepción y opinión del alumnado sobre las metodologías desarrolladas por el profesorado en las aulas de educación primaria. Así, el profesorado conocerá cómo es percibida su metodología por su alumnado y a partir de sus resultados identificará los aspectos que deben ser susceptibles de modificación, tomando consciencia de lo que planifica e implementa desde modelos centrados en el profesorado (de enseñanza) y no desde modelos más actuales centrados en el alumnado y en el desarrollo de sus competencias (de aprendizaje).

Así, el objetivo principal de este artículo es presentar el análisis de validación de una escala destinada a evaluar las estrategias de gestión e intervención docente o metodologías docentes para el nivel de educación primaria. Objetivo, que se consigue desde:

1. El diseño de la escala base de valoración de las metodologías docentes para alumnado de primaria.

2. La valoración del funcionamiento de dicha escala tras el estudio piloto realizado.

De forma específica, se presentan únicamente los resultados de validación de la escala, pero no olvidemos que para identificar elementos de mejora y la influencia de esta variable en el logro de las competencias del alumnado debería ser necesario desarrollar procesos de complementariedad de métodos en la valoración de ésta. Considerándose, así, necesarias otras alternativas o técnicas desde un enfoque cualitativo, como por ejemplo, la observación directa en el aula (Crespo, 2011) a través de plantillas de observación como la de Flanders (1970) o la observación participante de Oscar Guasch (1996), que permitan detectar situaciones de riesgo, detectar en qué medida se está adaptando el proceso de enseñanza a las necesidades del alumnado, etc.

\section{Método}

Este estudio se enmarca en procesos de revisión y elaboración de instrumentos de valoración o escalas de medida. Es decir, se parte de una propuesta inmersa en la medición educativa a través de:

1. Una aproximación cualitativa para la validación de contenido y de constructo, basada en comités de personas expertas, con la finalidad de identificar y revisar elementos relevantes en la valoración/evaluación de las metodologías docentes. 
2. Una aproximación cuantitativa, basada en un estudio de encuesta, para la validez de constructo. A partir del análisis métrico de la escala, la selección de ítems y el análisis de consistencia interna.

\subsection{Participantes}

Atendiendo a un tipo de muestreo no probabilístico incidental o por conveniencia trabajamos con un total de 9 centros de la Comunidad Valenciana, en el nivel de 6을 de Primaria, siete centros públicos y dos privados/concertados. Aunque dichos centros no fueron elegidos al azar y no son representativos de la población, sí concuerdan en características de la población de referencia como en tipo de centro (público y privados/concertados) y contexto geográfico y socioeconómico.

El total de estudiantes participantes fue de 324, con edades comprendidas entre 10 y 13 años y con una media de 11,28 años. En cuanto al sexo, destacar que el 50,6\% son chicos y el $49,4 \%$ son chicas. En cuanto a sus expectativas académicas relacionadas con dos materias instrumentales, señalar que el $91,6 \%$ de la muestra encuestada piensa que aprobará Matemáticas (cerca del $50 \%$ con sobresaliente o notable) y el 93,5\% que aprobarán la materia de Lengua.

\subsection{Variables e Instrumento}

El formato de aplicación para la recolección de información fue un instrumento diseñado ad hoc. Las variables que conforman el instrumento son de dos tipos:

a) Variables personales (características referidas al sexo y edad); variables de centro (nombre del centro, curso y grupo) y variables de rendimiento (años de permanencia en el colegio; curso repetido; nota esperada en Matemáticas y nota esperada en Lenguaje).

b) Variables tipo escala Likert sobre los aspectos considerados por los Comités de Expertos

y Expertas como pertinentes y adecuados respecto al constructo objeto de estudio, y que denominamos: Metodología docente.

\section{Resultados}

El proceso de análisis de las características métricas de la escala consistió en atender a la validez de contenido y de constructo, así como a la fiabilidad de los ítems (Chiva y Moral, 2009; Chiva y Ramos, 2011). 


\subsection{Diseño de la Escala (Validez de contenido y de constructo).}

Para garantizar la validez de la escala se lleva a cabo un proceso que se inicia con la búsqueda sistemática y rigurosa de investigaciones previas sobre la variable metodología docente. Esta revisión se realiza a través de distintas bases documentales en educación (ERIC; Catálogo de artículos de revistas sobre ciencias sociales y humanidades: ISOC; Red de Bases de Datos de Información Educativa del Ministerio de Educación y Ciencia: REDINED; Catálogo de Investigaciones Educativas del Ministerio de Educación y Ciencia: CIDE, entre otras).

En esta revisión se aprecia por un lado, la gran variedad de definiciones que existen (Azcarate, 1999; Casanova, 1999; Domenech, 1999 y Fernández March, 2006, entre otras), $y$, por otro, se constata que no se da un consenso a la hora de utilizar un mismo término, se habla de metodología de enseñanza, de estrategias metodológicas y de metodología docente. Asimismo, no hay una definición inclusiva, ni globalizadora de las diferentes perspectivas que pueden darse a este respecto. Por ello, a partir de las definiciones extraídas se define en este estudio metodología docente así: es el cómo enseñar y cómo ayudar a aprender, más concretamente son las estrategias de gestión e intervención docente (o pedagógica) que determinan las distintas maneras de enseñar en el aula con el objetivo de facilitar o guiar el aprendizaje de los y las estudiantes.

Una vez definido el constructo metodología docente se realiza el análisis de instrumentos y escalas diseñadas por otros investigadores e investigadoras que, bien directa o indirectamente, miden dicha variable (Monroy, Tanamachi y Backhoff, 2005; Muñoz, Ríos de Deus y Abalde, 2002; Solano, 2003). A partir de dicha revisión se establecen las siguientes especificaciones que definen la escala de medida posterior y su contenido.

- En primer lugar, se concretan ocho dimensiones básicas: Planificación del proceso de enseñanza/aprendizaje; Tipología actividades enseñanza/aprendizaje; Estilo docente; Utilización de los espacios y el tiempo de enseñanza; Estructura y Organización de los contenidos; Materiales curriculares y otros recursos didácticos; Sentido y papel de la evaluación; y Valoración general de la metodología docente.

- En segundo lugar, se exploran diversos instrumentos y se extrajeron un total de 234 ítems, los cuales a su vez son revisados a partir de los siguientes criterios: el concepto implícito del constructo, la edad de los sujetos y la adecuación de la formulación del 
enunciado. Después de esta revisión se reducen a 125 ítems compuestos ${ }^{3}$ con los cuales se diseña un cuestionario para la validación del comité de personas expertas, es decir, profesionales expertos en el tema (de la pedagogía y la psicopedagogía, profesorado de primaria, profesorado de secundaria y profesorado universitario). E objetivo de esta obtención de información se centra en extraer los ítems más significativos y relacionados con la variable estudiada atendiendo a: grado de relevancia de los elementos respecto a la evaluación de la metodología docente, grado de pertinencia de la información que aporta cada elemento, la adecuación a la fuente a la que queremos recoger la información y la adecuación al nivel educativo (primaria o secundaria).

Como resultado ${ }^{4}$, mencionar que los expertos y expertas valoran de forma positiva las ocho dimensiones planteadas para componer la escala (validez de constructo). Del mismo modo, valoran, de forma específica, los ítems en rangos positivos o muy positivos, con una homogeneidad bastante elevada. Aquí señalar que se eliminan o revisan los ítems que no alcanzaban ese nivel positivo y en los cuales existía bastante variabilidad en las respuestas aportada por los expertos/as (validez de contenido). Así y después de este análisis, del comité de expertos y expertas, se reducen los ítems: de los 125 ítems iniciales a 24 ítems compuestos. De este modo, se realiza el ajuste final del instrumento, adaptando cada ítem al tipo de audiencia al que va dirigido, alumnado de 6을 de primaria, con un formato adecuado y de fácil contestación. Por ello, se decide que los 24 ítems compuestos se descompongan en 102 ítems simples (donde se incluyen 8 ítems de identificación), utilizándose para su valoración una escala tipo Likert de 4 puntos, donde 1 equivale a nada/nunca, el 2 poco/casi nunca, el 3 mucho/muchas veces y el 4 totalmente/siempre.

${ }^{3}$ Definimos ítem compuesto aquel que tiene su origen en un diseño basado en facetas de GUTTMAN (Jornet y Suárez, 94). Ejemplo:

10. Frecuencia de uso de documentos para elaborar la programación de aula:

$\Rightarrow \quad$ Las programaciones didácticas de departamento.

$\Rightarrow \quad$ El libro de texto y sus guías didácticas

$\Rightarrow \quad$ Diversos libros de texto y sus guías didácticas...

${ }^{4}$ Los resultados concretos de la revisión de los expertos sobre la escala objeto de estudio aparecen detallados en Chiva, I. y Moral, A. (2009). 


\subsection{Análisis del funcionamiento de la Escala: Fiabilidad}

Para analizar el funcionamiento de la escala diseñada se presenta a continuación y en primer lugar, el análisis descriptivo de la misma con las respuestas ofrecidas por los 324 alumnos y alumnas de 6 p primaria. En segundo lugar, aparecen reflejados los resultados del análisis de consistencia interna de la escala.

En referencia al análisis que describe el comportamiento de los ítems y la opinión de los y las estudiantes, destacamos, en primer lugar, por dimensiones, los resultados más relevantes, atendiendo a los objetivos de este estudio.

En cuanto a la dimensión planificación del proceso de enseñanza/aprendizaje (dimensión compuesta por los ítems 9 y 10), hemos podido comprobar que en general, el alumnado percibe que el profesorado planifica mucho la actividad docente en el aula (ítem 10: $\bar{X}=3,23$ ), aunque es menos frecuente que les pida la opinión para esa planificación (ítem 9: $\bar{x}=2,42)$.

Si observamos los resultados de la dimensión tipología de actividades enseñanza/aprendizaje (compuesta por los ítems del 11 al 21 - ver Tabla 1- ), vemos que con una media de 2,99 es una de las dimensiones que mejores resultados ofrece. Al analizar de forma específica los ítems se aprecia que el ítem 19 tiene la media más alta $(\bar{X}=3,60)$, pues es donde los estudiantes muestran de forma abierta las actividades que más les gustan (realizar visitas fuera del centro, trabajar toda la clase junta...). A continuación, los ítems 13 y 14 ( $\bar{X}=3,42$ y $\bar{X}=3,36$ respectivamente) presentan una media de las más altas del conjunto, encontrándonos que las actividades que más les gustan a los chicos y chicas son trabajar con otro compañero o compañera y en equipo.

Por otro lado, el ítem 21 también destaca por su alta valoración y homogeneidad en la respuesta del grupo a la hora de contestar, constatada en su coeficiente de variación ${ }^{5}$ ( $\bar{X}=3,33 ; C . V .=21,3 \%)$, lo que supone que el alumnado se siente capaz de llevar a cabo las actividades que propone el profesorado. Por el contrario, lo que menos le gusta al alumnado son las actividades donde trabajan solos o son dirigidas por el profesorado (ítem 11: $\overline{\mathrm{X}}=2,43$ e ítem 12: $\bar{x}=2,38)$. Aunque es relevante destacar que en estos dos ítems el grupo no responde de forma similar $u$ homogénea, pues la variabilidad en la respuesta es media alta (45,3\% y $43,7 \%$ respectivamente).

\footnotetext{
${ }^{5}$ Para interpretar el coeficiente de variación de Pearson hemos tomado los siguientes valores: coeficiente $<33 \%$ significa que existe poca variabilidad en los datos y es una muestra muy compacta; coeficiente $>33 \%$ es una muestra muy dispersa.
} 
Tabla 1

Estadísticos Descriptivos: Dimensión Tipología actividades enseñanza-aprendizaje

\begin{tabular}{|c|c|c|c|c|}
\hline № & Enunciados Ítems & Media & $\begin{array}{l}\text { Desv. } \\
\text { Típica* }\end{array}$ & C.V. ${ }^{* *}$ \\
\hline ITEM11 & $\begin{array}{l}\text { Las actividades que me gustan más son aquellas en las que } \\
\text { puedo trabajar solo. }\end{array}$ & 2,43 & 1,10 & $45,27 \%$ \\
\hline ÍTEM12 & $\begin{array}{l}\text { Las actividades que me gustan más son aquellas en las que } \\
\text { puedo trabajar solo dirigido por el maestro o la maestra. }\end{array}$ & 2,38 & 1,04 & $43,7 \%$ \\
\hline ÍTEM13 & $\begin{array}{l}\text { Las actividades que me gustan más son aquellas en las que } \\
\text { puedo trabajar en equipo. }\end{array}$ & 3,42 & 0,74 & $21,64 \%$ \\
\hline ÍTEM14 & $\begin{array}{l}\text { Las actividades que me gustan más son aquellas en las que } \\
\text { puedo trabajar con otro compañero u otra compañera. }\end{array}$ & 3,36 & 0,82 & $24,4 \%$ \\
\hline ÍTEM15 & $\begin{array}{l}\text { Las actividades que me gustan más son aquellas en las que } \\
\text { hacemos debates. }\end{array}$ & 2,58 & 1,06 & $41,09 \%$ \\
\hline ÍTEM16 & $\begin{array}{l}\text { Las actividades que me gustan más son aquellas en las que } \\
\text { se exponen temas o trabajos en clase. }\end{array}$ & 3,01 & 0,90 & $29,9 \%$ \\
\hline ÍTEM17 & $\begin{array}{l}\text { Las actividades que me gustan más son aquellas en las que } \\
\text { realizamos trabajos de investigación y descubrimiento. }\end{array}$ & 2,88 & 1,09 & $37,85 \%$ \\
\hline ÍTEM18 & $\begin{array}{l}\text { Las actividades que me gustan más son aquellas en las que } \\
\text { salimos fuera del centro. }\end{array}$ & 3,29 & 0,88 & $26,75 \%$ \\
\hline ÍTEM19 & $\begin{array}{l}\text { Las actividades que me gustan más son aquellas en las } \\
\text { que.............. }\end{array}$ & 3,60 & 0,70 & $19,44 \%$ \\
\hline ÍTEM20 & $\begin{array}{l}\text { El maestro o la maestra suele adaptar las actividades en } \\
\text { función de nuestras necesidades e intereses. }\end{array}$ & 2,95 & 0,94 & $31,86 \%$ \\
\hline ÍTEM21 & $\begin{array}{l}\text { Me siento capaz de llevar a cabo todas las actividades que } \\
\text { me propone el maestro o la maestra en clase. }\end{array}$ & 3,33 & 0,71 & $21,32 \%$ \\
\hline
\end{tabular}

Fuente: Elaboración propia

En lo referente al estilo docente (dimensión conformada por los ítems del 22 al 55), apreciamos una media global de 2,92 - ver Tabla 2-.De forma específica, los ítems del 22 al 29 son los mejor valorados de la dimensión, es importante destacar que el alumnado cree que muchas veces el profesorado presta ayuda cuando lo necesitan (ítem 22), responde con precisión a las preguntas (ítem 24), les apoya en el trabajo que realizan (ítem 25), continua su explicación hasta que lo entienden (ítem 27) y les motiva a participar en las actividades de clase (ítem 29).

Por otro lado, el alumnado también destaca como positivo que el profesorado les premie cuando trabajan en equipo y colaboran entre ellos y ellas (ítem $31: \bar{x}=3,1$ ).

Respecto a las distintas opciones de cómo el profesorado castiga al alumnado (ítems del 47 al 54), cabe resaltar que, señalan que es más habitual que el profesorado les castigue riñéndoles delante de sus compañeros y compañeras (ítem 47) y a través de comunicaciones a las familias (ítem 53) que lo haga riñéndoles a solas (ítem 48), o expulsándolos de clase (ítem 52). Estos ítems miden en sentido contrario o inverso a la escala, al igual que ocurre con el ítem 23 "El maestro o la maestra sólo nos prepara para aprobar el examen", así lo positivo es que la media esté lo más cerca posible al punto 1 de la escala. 
Finalmente es para nosotras significativo señalar que el ítem 55 "Podemos preguntar todas las dudas en clase" es uno de los mejores valorados en la escala con una media de 3,70 , existiendo una importante homogeneidad en las respuestas dadas por el grupo (C.V.=18,1\%).

\section{Tabla 2}

Estadísticos Descriptivos: Dimensión Estilo docente

\begin{tabular}{|c|c|c|c|c|}
\hline № & Enunciados Ítems & Media & $\begin{array}{l}\text { Desv. } \\
\text { Típica* }\end{array}$ & C.V. ${ }^{* *}$ \\
\hline ÍTEM22 & $\begin{array}{l}\text { El maestro o la maestra presta ayuda adicional cuando } \\
\text { la necesitamos. }\end{array}$ & 3,54 & 0,78 & $22,03 \%$ \\
\hline ÍTEM23 & $\begin{array}{l}\text { El maestro o la maestra sólo nos prepara para aprobar } \\
\text { el examen. }\end{array}$ & 2,16 & 1,15 & $53,24 \%$ \\
\hline ÍTEM24 & $\begin{array}{l}\text { El maestro o la maestra responde con precisión a las } \\
\text { preguntas que le hacemos. }\end{array}$ & 3,37 & 0,78 & $23,15 \%$ \\
\hline ÍTEM25 & El maestro o la maestra apoya el trabajo que realizamos. & 3,31 & 0,85 & $25,68 \%$ \\
\hline ÍTEM26 & $\begin{array}{l}\text { El maestro o la maestra ayuda al alumnado en su } \\
\text { aprendizaje. }\end{array}$ & 3,66 & 0,61 & $16,67 \%$ \\
\hline ÍTEM27 & $\begin{array}{l}\text { El maestro o la maestra continúa su explicación hasta } \\
\text { que el alumnado lo entiende. }\end{array}$ & 3,68 & 0,64 & $17,39 \%$ \\
\hline ÍTEM28 & $\begin{array}{l}\text { El maestro o la maestra da al alumnado la oportunidad } \\
\text { de expresar sus opiniones. }\end{array}$ & 3,33 & 0,78 & $23,42 \%$ \\
\hline ÍTEM29 & $\begin{array}{l}\text { El maestro o la maestra nos motiva a participar en las } \\
\text { actividades de clase. }\end{array}$ & 3,35 & 0,76 & $22,69 \%$ \\
\hline ÍTEM30 & $\begin{array}{l}\text { El maestro o la maestra nos premia cuando nos } \\
\text { esforzamos en clase. }\end{array}$ & 3,03 & 0,98 & $32,34 \%$ \\
\hline ÍTEM31 & $\begin{array}{l}\text { El maestro o la maestra nos premia cuando trabajamos } \\
\text { en equipo y colaboramos con nuestros compañeros y } \\
\text { compañeras. }\end{array}$ & 3,08 & 1,00 & $32,47 \%$ \\
\hline ÍTEM32 & $\begin{array}{l}\text { El maestro o la maestra nos premia cuando respetamos } \\
\text { el trabajo de nuestros compañeros y compañeras. }\end{array}$ & 2,89 & 1,08 & $37,37 \%$ \\
\hline ÍTEM33 & $\begin{array}{l}\text { El maestro o la maestra nos premia cuando atendemos } \\
\text { durante las explicaciones. }\end{array}$ & 2,70 & 1,05 & $38,89 \%$ \\
\hline ÍTEM34 & $\begin{array}{l}\text { El maestro o la maestra nos premia cuando realizamos } \\
\text { las tareas y ejercicios para casa. }\end{array}$ & 2,91 & 1,09 & $37,46 \%$ \\
\hline ÍTEM35 & $\begin{array}{l}\text { El maestro o la maestra nos premia cuando cuidamos } \\
\text { materiales. }\end{array}$ & 2,75 & 1,15 & $41,82 \%$ \\
\hline ÍTEM36 & $\begin{array}{l}\text { El maestro o la maestra nos premia cuando hacemos } \\
\text { presentaciones/exposiciones de trabajos. }\end{array}$ & 3,01 & 1,02 & $33,89 \%$ \\
\hline ÍTEM37 & El maestro o la maestra nos premia cuando......... & 3,20 & 1,00 & $31,25 \%$ \\
\hline ÍTEM38 & $\begin{array}{l}\text { El maestro o la maestra nos felicita o recompensa } \\
\text { cuando actuamos correctamente en clase. }\end{array}$ & 3,08 & 0,97 & $31,49 \%$ \\
\hline ÍTEM39 & $\begin{array}{l}\text { El maestro o la maestra se enfada cuando no hacemos } \\
\text { los deberes que nos pone en clase }\end{array}$ & 3,14 & 0,91 & $28,98 \%$ \\
\hline ÍTEM40 & $\begin{array}{l}\text { El maestro o la maestra se enfada cuando no } \\
\text { participamos o bloqueamos los trabajos en equipo, } \\
\text { interrumpiendo a los demás. }\end{array}$ & 3,23 & 0,92 & $28,48 \%$ \\
\hline ÍTEM41 & $\begin{array}{l}\text { El maestro o la maestra se enfada cuando no } \\
\text { respetamos el trabajo de los compañeros y compañeras. }\end{array}$ & 3,16 & 0,99 & $31,33 \%$ \\
\hline ÍTEM42 & $\begin{array}{l}\text { El maestro o la maestra se enfada cuando no } \\
\text { atendemos durante las explicaciones o nos distraemos. }\end{array}$ & 3,13 & 0,92 & $29,39 \%$ \\
\hline ÍTEM43 & $\begin{array}{l}\text { El maestro o la maestra se enfada cuando no realizamos } \\
\text { las tareas o ejercicios para casa. }\end{array}$ & 3,08 & 0,96 & $31,17 \%$ \\
\hline ÍTEM44 & $\begin{array}{l}\text { El maestro o la maestra se enfada cuando no cuidamos } \\
\text { los materiales propios o de los compañeros y } \\
\text { compañeras. }\end{array}$ & 3,06 & 0,99 & $32,35 \%$ \\
\hline
\end{tabular}




\begin{tabular}{|c|c|c|c|c|}
\hline ÍTEM45 & $\begin{array}{l}\text { El maestro o la maestra se enfada cuando no realizamos } \\
\text { presentaciones/exposiciones adecuadas de trabajos. }\end{array}$ & 2,74 & 0,95 & $34,67 \%$ \\
\hline ÍTEM46 & $\begin{array}{l}\text { El maestro o la maestra se enfada } \\
\text { cuando.......................... }\end{array}$ & 3,48 & 0,82 & $23,56 \%$ \\
\hline ÍTEM47 & $\begin{array}{l}\text { El maestro o la maestra nos castiga riñéndonos delante } \\
\text { de los compañeros y compañeras. }\end{array}$ & 2,72 & 0,96 & $35,29 \%$ \\
\hline ÍTEM48 & El maestro o la maestra nos castiga riñéndonos a solas. & 2,05 & 0,93 & $45,37 \%$ \\
\hline ÍTEM49 & $\begin{array}{l}\text { El maestro o la maestra nos castiga a través de } \\
\text { anotaciones en nuestros trabajos o cuadernos. }\end{array}$ & 2,34 & 1,11 & $47,44 \%$ \\
\hline ÍTEM50 & $\begin{array}{l}\text { El maestro o la maestra nos castiga a través trabajos } \\
\text { extras. }\end{array}$ & 1,78 & 0,94 & $52,81 \%$ \\
\hline ÍTEM51 & $\begin{array}{l}\text { El maestro o la maestra nos castiga mandándonos a ver } \\
\text { al tutor o tutora, orientador u orientadora o director o } \\
\text { directora del centro. }\end{array}$ & 1,68 & 0,87 & $51,79 \%$ \\
\hline ÍTEM52 & $\begin{array}{l}\text { El maestro o la maestra nos castiga expulsándonos de } \\
\text { clase. }\end{array}$ & 1,99 & 0,94 & $47,24 \%$ \\
\hline ÍTEM53 & $\begin{array}{l}\text { El maestro o la maestra nos castiga a través de } \\
\text { comunicaciones a la familia. }\end{array}$ & 2,46 & 0,98 & $39,84 \%$ \\
\hline ÍTEM54 & El maestro o la maestra nos castiga...$\ldots \ldots \ldots \ldots \ldots \ldots \ldots$ & 3,09 & 0,91 & $29,45 \%$ \\
\hline ÍTEM55 & Podemos preguntar todas las dudas en clase. & 3,70 & 0,67 & $18,11 \%$ \\
\hline
\end{tabular}

Fuente: Elaboración propia.

En relación con la utilización de los espacios y el tiempo (dimensión conformada por los ítems del 56 al 62 de la escala) vemos que son elementos valorados con una media tendente al poco o casi nunca lo hace así el profesorado $(\overline{\mathrm{X}}=2,5)$. Si se analizan los ítems ver Tabla 3- nos encontramos con que el alumnado opina que la distribución de mesas y sillas favorece la comunicación en clase (ítem 56: $\overline{\mathrm{x}}=2,99$ ). En cuanto a lo relacionado con cómo están más cómodos respecto a la colocación de las mesas en el aula (ítems del 57 al 62), señalar que la distribución mejor valorada es la de tener las mesas en pequeño grupo (ítem 60) y la peor en forma de circulo (ítem 59), aunque es importante apuntar que existe una heterogeneidad en la opinión dada por los chicos y chicas respecto a todos estos contenidos. 
Tabla 3

Estadísticos Descriptivos: Dimensión Utilización de los espacios y del tiempo

\begin{tabular}{|c|c|c|c|c|}
\hline № & Enunciados Ítems & Media & $\begin{array}{l}\text { Desv. } * \\
\text { Típica }\end{array}$ & C. $V_{.}^{* *}$ \\
\hline ÍTEM56 & $\begin{array}{l}\text { La distribución de mesas y sillas en clase favorece que todos } \\
\text { y todas nos comuniquemos fácilmente. }\end{array}$ & 2,99 & 1,01 & $33,78 \%$ \\
\hline ÍTEM57 & $\begin{array}{l}\text { Estamos más cómodos/as y aprendemos más si las mesas } \\
\text { están unidas en líneas / filas. }\end{array}$ & 2,55 & 1,17 & $45,88 \%$ \\
\hline ÍTEM58 & $\begin{array}{l}\text { Estamos más cómodos/as y aprendemos más si las mesas } \\
\text { están de forma individual. }\end{array}$ & 2,19 & 1,19 & $54,34 \%$ \\
\hline ÍTEM59 & $\begin{array}{l}\text { Estamos más cómodos/as y aprendemos más si las mesas } \\
\text { están en forma de círculo. }\end{array}$ & 1,98 & 1,11 & $56,06 \%$ \\
\hline ÍTEM60 & $\begin{array}{l}\text { Estamos más cómodos/as y aprendemos más si las mesas } \\
\text { están en pequeño grupo. }\end{array}$ & 2,69 & 1,13 & $42,01 \%$ \\
\hline ÍTEM61 & $\begin{array}{l}\text { Estamos más cómodos/as y aprendemos más si las mesas } \\
\text { están en forma de } U \text {. }\end{array}$ & 2,42 & 1,21 & $50,00 \%$ \\
\hline ÍTEM62 & $\begin{array}{l}\text { Estamos más cómodos/as y aprendemos más si las mesas } \\
\text { están........ }\end{array}$ & 3,45 & 0,86 & $24,93 \%$ \\
\hline
\end{tabular}

Fuente: Elaboración propia

La dimensión estructura y organización de los contenidos la forman los ítems del 63 al 68 - ver Tabla 4-. La media de esta dimensión es de 2,9 lo que indica que, en general, el alumnado está de acuerdo con la estructura y organización de los contenidos. Es habitual que el profesorado al comenzar el tema exponga los objetivos a alcanzar (ítem 63), explique el tema nuevo, haga ejercicios (ítem 65) y que busque ejemplos de la vida diaria (ítem 66). Sin embargo, es menos habitual que el profesorado repase los conceptos del tema anterior (ítem 64) y que entregue material adicional relacionado con el tema (67).

Tabla 4

Estadísticos Descriptivos: Dimensión Estructura y Organización de contenidos

\begin{tabular}{|l|l|c|c|c|}
\multicolumn{2}{|c|}{ № } & \multicolumn{1}{|c|}{ Media } & \multicolumn{1}{|c|}{$\begin{array}{c}\text { Desv. } \\
\text { Típica* }\end{array}$} & C.V.** \\
\hline ÍTEM63 & $\begin{array}{l}\text { Normalmente cuando el maestro o la maestra comienza un } \\
\text { tema nuevo suele exponer objetivos que pretenden alcanzar. }\end{array}$ & 3,12 & 1,02 & $32,69 \%$ \\
\hline ÍTEM64 & $\begin{array}{l}\text { Normalmente cuando el maestro o la maestra comienza un } \\
\text { tema nuevo suele repasar los conceptos de temas anteriores. }\end{array}$ & 2,54 & 1,01 & $39,76 \%$ \\
\hline ÍTEM65 & $\begin{array}{l}\text { Normalmente cuando el maestro o la maestra comienza un } \\
\text { tema nuevo suele explicar el tema de modo completo, para } \\
\text { luego hacer ejercicios. }\end{array}$ & 3,09 & 1,00 & $32,36 \%$ \\
\hline ÍTEM66 & $\begin{array}{l}\text { Normalmente cuando el maestro o la maestra comienza un } \\
\text { tema nuevo suele buscar ejemplos de la vida diaria que } \\
\text { faciliten la explicación. }\end{array}$ & 2,93 & 0,97 & $33,11 \%$ \\
\hline ÍTEM67 & $\begin{array}{l}\text { Normalmente cuando el maestro o la maestra comienza un } \\
\text { tema nuevo suele entregar al alumnado material relacionado } \\
\text { con el tema. }\end{array}$ & 2,37 & 1,07 & $45,15 \%$ \\
\hline ÍTEM68 & $\begin{array}{l}\text { Normalmente cuando el maestro o la maestra comienza un } \\
\text { tema nuevo suele............. }\end{array}$ & 3,51 & 0,84 & $23,93 \%$ \\
\hline $\begin{array}{l}\text { *Desv. Típ. = Desviación típica } \\
\text { **C.V. }=\text { Coeficiente de Variación }\end{array}$ & & & \\
\hline
\end{tabular}

Fuente: Elaboración propia 
En cuanto a los materiales curriculares y otros recursos didácticos (ítems del 69 al 79) - ver Tabla 5- hemos podido apreciar que el profesorado utiliza el libro de texto para dar sus clases (ítem 69), la pizarra (ítem 70), el material elaborado por ellos mismos (ítem 75) y los materiales impresos editados (ítem 77). No obstante, no es tan habitual que utilice los libros de la biblioteca (ítem 71), la prensa escrita (ítem 72), los materiales audiovisuales (ítem 73 y 74) o material manipulativo (ítem 78). Podemos destacar que los ítems que presentan valoraciones más altas tienen menor dispersión y mayor homogeneidad en la respuesta de los estudiantes.

Tabla 5

Estadísticos Descriptivos: Dimensión Materiales curriculares y otros recursos didácticos

\begin{tabular}{|c|c|c|c|c|}
\hline № & Enunciados Ittems & Media & $\begin{array}{l}\text { Desv. } \\
\text { Típica* }\end{array}$ & C.V. ${ }^{* *}$ \\
\hline ITEM69 & $\begin{array}{l}\text { El maestro o la maestra utiliza el libro de texto como única } \\
\text { guía para dar las clases. }\end{array}$ & 2,60 & 0,94 & $36,15 \%$ \\
\hline ÍTEM70 & $\begin{array}{l}\text { Es habitual que en clase se utilicen apuntes de la pizarra o } \\
\text { encerado. }\end{array}$ & 3,14 & 0,91 & $28,98 \%$ \\
\hline ÍTEM71 & $\begin{array}{l}\text { Es habitual que en clase se utilicen libros de la biblioteca del } \\
\text { colegio o del aula. }\end{array}$ & 2,54 & 1,12 & $44,09 \%$ \\
\hline ÍTEM72 & Es habitual que en clase se utilicen prensa escrita. & 1,92 & 0,89 & $46,35 \%$ \\
\hline ÍTEM73 & Es habitual que en clase se utilicen los ordenadores. & 2,04 & 0,98 & $48,04 \%$ \\
\hline ÍTEM74 & Es habitual que en clase se utilicen medios audiovisuales. & 2,19 & 0,90 & $41,10 \%$ \\
\hline ÍTEM75 & $\begin{array}{l}\text { Es habitual que en clase se utilicen materiales elaborados por } \\
\text { el propio profesorado. }\end{array}$ & 3,00 & 0,90 & $30,00 \%$ \\
\hline ÍTEM76 & $\begin{array}{l}\text { Es habitual que en clase se utilicen materiales elaborados en } \\
\text { equipo (elaborados por el profesorado). }\end{array}$ & 2,51 & 0,94 & $37,45 \%$ \\
\hline ÍTEM77 & $\begin{array}{l}\text { Es habitual que en clase se utilicen materiales impresos } \\
\text { editados. }\end{array}$ & 2,99 & 0,89 & $29,77 \%$ \\
\hline ÍTEM78 & $\begin{array}{l}\text { Es habitual que en clase se utilicen material manipulativo (de } \\
\text { laboratorio, juegos, instrumentos...). }\end{array}$ & 2,20 & 1,00 & $45,45 \%$ \\
\hline ÍTEM79 & Es habitual que en clase se utilicen................... & 3,33 & 0,92 & $27,63 \%$ \\
\hline
\end{tabular}

Fuente: Elaboración propia

Si analizamos las opiniones de los estudiantes sobre el sentido y papel de la evaluación (ítems del 80 al 101) -ver Tabla 6-, comprobamos que los ítems mejor valorados con una puntuación superior a 3,5 puntos, son: el ítem 93 e ítem 95, en ellos el alumnado destaca que lo más importante para sacar buenas calificaciones es hacer los deberes y sacar buenas notas en los controles. Por otro lado, los ítems peor valorados son el 100 y el 87 ( $\bar{X}=2,16$ y $\bar{X}=2,26$ respectivamente). Esto significa que los estudiantes son más críticos a la hora de valorar si el maestro/a les motiva cuando obtienen puntuaciones bajas y si les evalúa a través de la nota que cada uno se pone en los ejercicios y controles. 
Tabla 6

Estadísticos Descriptivos: Dimensión Sentido y papel de la evaluación

\begin{tabular}{|c|c|c|c|c|}
\hline № & ciados Ítems & Media & $\begin{array}{l}\text { Desv. } \\
\text { Típica* }\end{array}$ & C.V. ${ }^{* *}$ \\
\hline ITEM80 & $\begin{array}{l}\text { El maestro o la maestra nos informa de los criterios de } \\
\text { evaluación que después aplica. }\end{array}$ & 2,95 & 0,95 & $32,20 \%$ \\
\hline ÍTEM81 & $\begin{array}{l}\text { El maestro o la maestra nos evalúa a través de controles } \\
\text { orales. }\end{array}$ & 2,39 & 0,97 & $40,59 \%$ \\
\hline ÍTEM82 & $\begin{array}{l}\text { El maestro o la maestra nos evalúa a través de controles } \\
\text { escritos. }\end{array}$ & 3,45 & 0,92 & $26,67 \%$ \\
\hline ÍTEM83 & $\begin{array}{l}\text { El maestro o la maestra nos evalúa a través de controles en } \\
\text { los que podemos utilizar material. }\end{array}$ & 2,32 & 1,02 & $43,97 \%$ \\
\hline ÍTEM84 & $\begin{array}{l}\text { El maestro o la maestra nos evalúa a través de observarnos } \\
\text { cuando trabajamos en clase. }\end{array}$ & 2,88 & 1,01 & $35,07 \%$ \\
\hline ÍTEM85 & $\begin{array}{l}\text { El maestro o la maestra nos evalúa a través de ejercicios o } \\
\text { actividades que hemos hecho en el cuaderno. }\end{array}$ & 3,07 & 0,96 & $31,27 \%$ \\
\hline ÍTEM86 & $\begin{array}{l}\text { El maestro o la maestra nos evalúa a través de la corrección } \\
\text { de los deberes. }\end{array}$ & 3,07 & 1,02 & $33,22 \%$ \\
\hline ÍTEM87 & $\begin{array}{l}\text { El maestro o la maestra nos evalúa a través de la nota que } \\
\text { nosotros nos ponemos en ejercicios/controles. }\end{array}$ & 2,26 & 1,04 & $46,02 \%$ \\
\hline ÍTEM88 & El maestro o la maestra nos evalúa a través de. & 3,49 & 0,75 & $21,49 \%$ \\
\hline ÍTEM89 & $\begin{array}{l}\text { Nuestro maestro o maestra hace un seguimiento personal de } \\
\text { cada alumno/a. }\end{array}$ & 2,70 & 1,11 & $41,11 \%$ \\
\hline ÍTEM90 & $\begin{array}{l}\text { La evaluación final es fruto del trabajo realizado a lo largo de } \\
\text { todo el curso no sólo de los exámenes. }\end{array}$ & 3,53 & 0,82 & $23,23 \%$ \\
\hline ÍTEM91 & $\begin{array}{l}\text { Nuestro maestro o maestra explica la evaluación revisándola } \\
\text { si considera que puede haber un error. }\end{array}$ & 3,02 & 0,99 & $32,78 \%$ \\
\hline ÍTEM92 & $\begin{array}{l}\text { Para sacar buenas calificaciones en mi clase es importante } \\
\text { portarse bien. }\end{array}$ & 3,48 & 0,78 & $22,41 \%$ \\
\hline ÍTEM93 & $\begin{array}{l}\text { Para sacar buenas calificaciones en mi clase es importante } \\
\text { hacer los deberes. }\end{array}$ & 3,61 & 0,65 & $18,01 \%$ \\
\hline ÍTEM94 & $\begin{array}{l}\text { Para sacar buenas calificaciones en mi clase es importante } \\
\text { hacer bien las actividades y trabajos. }\end{array}$ & 3,47 & 0,74 & $21,33 \%$ \\
\hline ÍTEM95 & $\begin{array}{l}\text { Para sacar buenas calificaciones en mi clase es importante } \\
\text { sacar buenas notas en los controles. }\end{array}$ & 3,56 & 0,71 & $19,94 \%$ \\
\hline ÍTEM96 & $\begin{array}{l}\begin{array}{l}\text { Para sacar buenas calificaciones en } \\
\text { importante................. }\end{array} \\
\text { mi clase es }\end{array}$ & 3,62 & 0,72 & $19,89 \%$ \\
\hline ÍTEM97 & $\begin{array}{l}\text { Cuando el alumnado obtiene calificaciones muy bajas } \\
\text { nuestro maestro o maestra habla con él/ella. }\end{array}$ & 3,06 & 0,97 & $31,70 \%$ \\
\hline ÍTEM98 & $\begin{array}{l}\text { Cuando el alumnado obtiene calificaciones muy bajas } \\
\text { nuestro maestro o maestra suele darle tareas adicionales. }\end{array}$ & 2,38 & 1,03 & $43,28 \%$ \\
\hline ÍTEM99 & $\begin{array}{l}\text { Cuando el alumnado obtiene calificaciones muy bajas } \\
\text { nuestro maestro o maestra suele avisar a los padres y } \\
\text { madres. }\end{array}$ & 2,81 & 1,09 & $38,79 \%$ \\
\hline ITTEM100 & $\begin{array}{l}\text { Cuando el alumnado obtiene calificaciones muy bajas } \\
\text { nuestro maestro o maestra suele tratar de motivarle dándole } \\
\text { privilegios o cargos especiales (aux. profes.). }\end{array}$ & 2,16 & 1,01 & $46,76 \%$ \\
\hline ÍTEM101 & $\begin{array}{l}\text { Cuando el alumnado obtiene calificaciones muy bajas } \\
\text { nuestro maestro o maestra suele } \ldots \ldots \ldots \ldots \ldots \ldots \ldots \ldots \ldots \ldots\end{array}$ & 3,42 & 0,85 & $24,85 \%$ \\
\hline
\end{tabular}

Fuente: Elaboración propia 
Por último, y en cuanto a la valoración general, ítem 102, con una media de 3,49 y homogeneidad en las valoraciones dadas al mismo (C.V.=23,21\%), el grupo de estudiantes encuestados piensa que la forma de dar la clase por parte del profesorado es adecuada para su aprendizaje.

Como resultados generales de este análisis podemos destacar que la media total del instrumento supera la valoración media de la escala utilizada $(\bar{X}=2,89)$. De forma concreta y respecto a las dimensiones de la escala, destacar que la dimensión mejor valorada es "Valoración general de la metodología docente empleada" ( $\bar{X}=3,50)$, seguida de "Tipología de actividades de enseñanza/aprendizaje" $(\bar{x}=2,99)$ y del "Sentido y Papel de la evaluación" $(\bar{X}=2,98)$, mientras que las peores valoradas son "Materiales curriculares y otros recursos" y la "Utilización de espacios y tiempo", con una media de 2,52 en ambas dimensiones -ver Tabla 7-. En cuanto a la variabilidad de las respuestas u opiniones del conjunto de estudiantes, podemos destacar que existe homogeneidad en ellas, ya que todas las dimensiones presentan un coeficiente de variación menor del 33\% -ver Tabla No. 7-, lo que supone que las medias de las valoraciones son representativas en todas ellas. Aunque sí que podemos observar una mayor homogeneidad en la opinión de los estudiantes cuando valoran las dimensiones relacionadas con "Estilo docente" (12,3\%), "Tipología de actividades" $(13,4 \%)$ y "Sentido y papel de la evaluación" $(16,1 \%)$.

Tabla 7

Estadísticos descriptivos Dimensiones Escala metodología docente \begin{tabular}{|l|c|l|l|l|l|l|}
\hline DIMENSIONES ESCALA & $N$ & Mínimo & Máximo & Media & Desv. tip* & C.V.**
\end{tabular}

\begin{tabular}{|l|c|c|c|c|c|c|}
\hline Planificación proceso E/A & 323 & 1,00 & 4,00 & 2,83 & 0,72 & $25,44 \%$ \\
\hline Tipología actividades E/A & 324 & 1,90 & 3,82 & 2,99 & 0,40 & $13,38 \%$ \\
\hline Estilo docente & 324 & 1,87 & 3,74 & 2,92 & 0,36 & $12,33 \%$ \\
\hline Utilización espacios y tiempo & 324 & 1,00 & 4,00 & 2,52 & 0,51 & $20,24 \%$ \\
\hline $\begin{array}{l}\text { Estructura y organización de } \\
\text { contenidos }\end{array}$ & 324 & 1,20 & 4,00 & 2,85 & 0,65 & $22,80 \%$ \\
\hline $\begin{array}{l}\text { Materiales Curriculares y otros } \\
\text { recursos }\end{array}$ & 322 & 1,22 & 4,00 & 2,52 & 0,56 & $22,22 \%$ \\
\hline Sentido y Papel de la Evaluación & 321 & 1,16 & 3,84 & 2,98 & 0,48 & $16,11 \%$ \\
\hline Valoración General & 315 & 1,00 & 4,00 & 3,50 & 0,81 & $23,14 \%$ \\
\hline Media total de la Escala & 324 & 1,82 & 3,55 & 2,89 & 0,37 & $12,80 \%$ \\
\hline $\begin{array}{l}{ }^{*} \text { Desv. Típ. = Desviación típica } \\
\text { **C.V. = Coeficiente de Variación }\end{array}$
\end{tabular}

Fuente: Elaboración propia 


\subsection{Análisis consistencia interna de la escala}

El análisis de consistencia interna del instrumento se realiza sobre 84 de los 102 ítems incluidos y sobre los 324 casos/sujetos. Para este análisis, por un lado, se han omitido los 8 ítems iniciales de identificación y las valoraciones de las preguntas abiertas de la escala dado que muchos sujetos omiten la respuesta y, por tanto, su valoración (ítems 19, 37, 46, $54,62,68,79,88,96,101)$. Por otro lado, se han sustituido los valores perdidos de los ítems de la escala por la mediana ${ }^{6}$ de cada ítem. Esto es necesario para que en el análisis de consistencia no se eliminen más de la mitad de los casos y así poder realizar el análisis sobre los 324 casos/sujetos.

Específicamente en este análisis de consistencia interna, para estimar la fiabilidad de la escala, se utiliza el coeficiente Alfa de Cronbach, obteniéndose en este caso una fiabilidad alta $(\alpha=0,906)$, lo que indica que el conjunto de ítems seleccionados miden de forma coherente y consistente - ver Tabla 8-.

Tabla 8

Estadísticos de fiabilidad de la Escala

\begin{tabular}{|c|c|c|}
\hline Alfa de Cronbach & $\begin{array}{c}\text { Alfa de Cronbach basada en } \\
\text { los elementos tipificados }\end{array}$ & N de elementos \\
\hline 0,906 & 0,909 & 84 \\
\hline
\end{tabular}

Fuente: Elaboración propia

Además de la fiabilidad total de la escala se calculan los estadísticos de fiabilidad para los elementos que la componen, con la finalidad de analizar el poder de discriminación de cada uno de ellos y realizar de acuerdo con ello la selección de los mismos. Los criterios establecidos para el análisis de discriminación de los ítems fueron los siguientes:

a) En la correlación elemento- total corregida, se fijó como criterio eliminar los ítems cuya correlación con el resto del instrumento era inferior a 0.20 o negativa, revisar aquellos que están entre 0.20 y 0,40 y mantener los superiores a 0,40;

b) Respecto al estadístico de alfa de Cronbach si se elimina el elemento, se decide eliminar aquellos ítems que no mejoran la fiabilidad de la escala analizada, revisar aquellos en los que se mantiene la misma fiabilidad y mantener los que una vez eliminados disminuyen la fiabilidad de la escala.

\footnotetext{
${ }^{6}$ Se ha optado por la mediana por las características métricas de las variables.
} 
A continuación se presenta una tabla resumen donde se reflejan los ítems a revisar y a eliminar de acuerdo con este análisis y los criterios establecidos - ver Tabla 9-.

En total, se eliminan 19 ítems pertenecientes en su mayoría a las dimensiones de tipología de actividades (ítems 11, 12, 13 y 21), estilo docente (ítems 23, 39, 43, 47, 49, 50, 51,52 ), utilización de espacios (ítems 57, 58, 59, 60 y 61), materiales (ítem 69) y evaluación (ítem 100). Asimismo, un total de 32 ítems deberían ser revisados.

\section{Tabla 9}

Ítems a revisar y a eliminar de la escala

\begin{tabular}{|c|c|c|}
\hline & Ítems a revisar & Ítems a eliminar \\
\hline $\begin{array}{l}\text { Según } \\
\text { elemento-total corregida }\end{array}$ & $\begin{array}{l}9,10,14,15,16,17,18,22, \\
24,27,40,41,42,44,45,48, \\
53,55,56,64,65,70,72,74, \\
77,82,83,87,90,92,93,95\end{array}$ & $\begin{array}{l}11,12,13,21,23,39,43 \\
47,49,50,51,52,57,58 \\
59,60,61,69,100\end{array}$ \\
\hline $\begin{array}{l}\text { Según Alfa de Cronbach si } \\
\text { se elimina el elemento }\end{array}$ & $12,43,49,50,51,60,61,69$ & $\begin{array}{l}11,13,23,39,47,52,57 \\
58,59\end{array}$ \\
\hline Ambos Estadísticos & $\begin{array}{l}9,10,14,15,16,17,18,22, \\
24,27,40,41,42,44,45,48, \\
53,55,56,64,65,70,72,74, \\
77,82,83,87,90,92,93,95\end{array}$ & $\begin{array}{l}11,12,13,21,23,39,43 \\
47,49,50,51,52,57,58 \\
59,60,61,69,100\end{array}$ \\
\hline
\end{tabular}

Fuente: Elaboración propia.

\section{Conclusiones}

En este estudio la estrategia de análisis seguida ha intentado responder al objetivo principal "presentar el análisis de validación de una escala destinada a evaluar las metodologías docentes o, lo que es lo mismo, las estrategias de gestión e intervención en el aula para el nivel de Educación Primaria".

Respecto al instrumento diseñado y los resultados obtenidos en la revisión documental y de expertos, señalar que en general los expertos/as valoran los ítems y dimensiones en rangos positivos o muy positivos, con una homogeneidad de respuesta/opinión bastante elevada.

Respecto a la revisión empírica realizada, indicar que la percepción que el alumnado tiene en general de la metodología docente desarrollada por el profesorado en su aula supera el punto medio de la escala, alcanzando el 2,88. De este modo, las dimensiones mejor valoradas son la de "valoración general de los alumnos/as respecto a la metodología docente empleada" $(\overline{\mathrm{X}}=3,49)$, seguida por "Tipología de actividades de enseñanza/aprendizaje" ( $\overline{\mathrm{X}}=2,98)$ y por el "Papel de la evaluación" $(\overline{\mathrm{X}}=2,97)$. Por otro lado, 
los ítems peores valorados son aquellos que hacen referencia a los "Materiales curriculares $y$ otros recursos" y a la "Utilización de espacios y tiempo" con una media de 2,52.

En lo referente al análisis de consistencia interna y selección de elementos, destacar que tras su cálculo $(\alpha=0,906)$ se obtiene información relevante que permite tomar decisiones sobre los ítems a eliminar, revisar o mantener en la escala de metodología docente y realizar una propuesta de escala, tal y como aparece construida en el Anexo 1.

De esta forma, al asumir los matices que se han ido comentando en este artículo y al creer en la necesidad de llevar a cabo una revisión constante de las escalas destinadas a medir estos constructos, como conclusión de este estudio, podemos presentar una escala para la valoración de la variable metodologías docentes en educación primaria. Esta escala está conformada por 65 ítems simples y 8 dimensiones - ver Tabla No.10 en Anexo 1-. Asimismo, creemos conveniente medir dicha valoración a través de una escala Likert de cuatro puntos para favorecer el posicionamiento de los alumnos/as (desde (1) Muy en desacuerdo al (4) Muy de acuerdo o desde (1) Nunca a (4) Siempre).

A partir de estos resultados y siendo conscientes de que, desde las distintas normativas educativas actuales, se busca un conocimiento y reflexión sobre la práctica educativa, la comprensión del proceso educativo de forma contextualizada y una aproximación mayor al educando y a la comunidad (Tejada, 2011) pensamos que es de gran utilidad, en este contexto, presentar esta escala de evaluación de las estrategias de gestión e intervención docente o metodologías docente. Una escala que, a nuestro modo de entender, puede permitir al profesorado de educación primaria ser consciente de:

- los puntos fuertes (serían todos aquellos ítems o dimensiones con valoraciones medias que se sitúen en el intervalo 3 y $4^{7}$ ) y

- los puntos débiles (serían todos aquellos ítems o dimensiones con valoraciones medias que se sitúen en el intervalo entre 1 y 2), de estos puntos débiles surgirán los aspectos a mejorar de su metodología docente ${ }^{8}$ - ver Anexo 1-.

Además, debe señalarse que obtener valoraciones altas en esta escala hace referencia a la utilización de unas estrategias de gestión e intervención en el aula adecuadamente

\footnotetext{
${ }^{7}$ Hemos de tener en cuenta que en la escala propuesta, Anexo 1, los ítems 29 y 30 tienen valoraciones inversas al resto, por lo que sería necesario invertir sus valoraciones para obtener los puntos fuertes, puntos débiles y aspectos a mejorar de la misma.

${ }^{8}$ Para desarrollar el cálculo de la información aportada por un grupo de estudiantes, el profesor/a puede realizar los siguientes procedimientos: calculo valoración media cada ítem, calculo valoración media por dimensiones y/o calculo valoración media total de la escala.
} 
planificadas y organizadas, es decir, caracterizadas por: tener en cuenta las aportaciones de los estudiantes, utilizar una gran variedad de actividades, materiales y recursos educativos, servir de apoyo al alumnado, atender su ritmo de aprendizaje, usar de forma adecuada los espacios y los tiempos, estructurar y organizar los contenidos basándose en el aprendizaje significativo y constructivo y ofrecer procedimientos e instrumentos de evaluación diversos para el aprendizaje continuo.

En conclusión al igual que Sánchez (2011), concebimos el proceso de enseñanzaaprendizaje como una secuencia que, partiendo de las capacidades, necesidades e intereses de los estudiantes plantee estrategias metodológicas que se sitúen en el aprendizaje significativo y contribuya al desarrollo las competencias necesarias para llegar a ser ciudadanos/as autónomos/as, reflexivos/as, críticos/as y constructivos/as.

Por último, señalar que con este estudio se han establecido premisas que justifican la continuación de investigaciones que ayuden a conocer las metodologías docentes de cada una de las aulas de primaria. Es decir, mediante la escala final resultante - ver Anexo 1podremos conocer mejor cómo se trabaja día a día en el aula, información que nos permitirá mejorar y adaptar la metodología docente a las necesidades del alumnado para un funcionamiento particular y diferenciador de su rendimiento académico.

\section{Referencias}

Ademar, Horacio y Ariel, Sebastián (Coords). (2008). De aprendizajes, competencias y capacidades en la educación primaria. Desandando caminos para construir nuevos senderos. Revista Iberoamericana de Educación, (47/3), 1-13.

Arribas, José María. (2012). El rendimiento académico en función del sistema de evaluación empleado. RELIEVE, 18(1). Recuperado de http://www.uv.es/RELIEVE/v18n1/RELIEVEv18n1 3.htm

Azcarate, María del Pilar. (1999). Metodología de enseñanza. Cuadernos de Pedagogía, 276.

Bermejo, Vicente. (2006). Formación del profesorado y cambios metodológicos. Didáctica, Innovación y Multimedia, 3, 1-7.

Carrera, Celia y Marín, Rigoberto. (2011). Modelo pedagógico para el desarrollo de competencias en educación superior. Revista Actualidades Investigativas en Educación, 11(1), 1-32. 1 Recuperado http://revista.inie.ucr.ac.cr/index.php/aie/article/view/490

Casanova, María Antonia. (1999). Manual de Evaluación Educativa. Madrid: La Muralla. 
Chiva, Inmaculada y Moral, Ana María. (2009). Diseño y revisión lógica de una escala para evaluar la metodología docente en primaria y secundaria. Huelva: AIDIPE.

Chiva, Inmaculada y Ramos, Genoveva. (2011). Revisión Empírica de una Escala para evaluar la Metodología Docente en Educación Secundaria. Madrid: AIDIPE

Crespo, Julia María. (2011). El educador competente. En Isabel Cantón y Margarita PinoJuste (Coords), Diseño y desarrollo curricular (pp.41-56). Madrid: Alianza Editorial

De Miguel, Mario. (2006). Metodologías de enseñanza-aprendizaje para el desarrollo de competencias: orientaciones para el profesorado universitario ante el Espacio Europeo de Educación Superior. Madrid: Alianza.

Domenech, Fernando. (1999). El proceso de enseñanza/aprendizaje universitario. Castelló: Universitat Jaume I.

Fernández March, Amparo. (2006). Metodologías activas para la formación de competencias. Educatio Siglo XXI, 24. Recuperado de http://revistas.um.es/educatio/article/view/152

Flanders, Ned. (1970). Análisis de interacción didáctica. Madrid: Anaya.

Garrote, Daniel. (2008). Nuevos retos educativos: el modelo docente en el espacio europeo. Contextos educativos, 11, 246.

Guasch, Oscar. (1996). Observación participante. Centro de Investigaciones Sociológicas (CIS).

Jornet, Jesús Miguel y Backhoff, Eduardo. (2004). Análisis Diferencial de perfiles de rendimiento y variables asociadas en los proyectos mexicanos EXANI-I, TIMMS y PISA. En Felipe Tirado (coord.), Evaluación de la educación en México. Indicadores de EXANI-I (pp. 423-482). Editorial: México: CENEVAL

Lira, Rosa Inés. (2010). Las metodologías activas y el foro presencial: su contribución al desarrollo del pensamiento crítico. Revista Actualidades Investigativas en Educación, 10(1), 1-18. Recuperado de http://revista.inie.ucr.ac.cr/index.php/aie/article/view/420

MEC (2006). Real Decreto 1513/2006, de 7 de diciembre, por el que se establecen las enseñanzas mínimas de Educación Primaria, BOE-A-2006-21409.C.F.R.

Mendoza, Michelle y Covarrubias, Carmen Gloria. (2014). Competencias profesionales movilizadas en el prácticum: percepciones del estudiantado del grado de maestro de educación primaria. Revista Actualidades Investigativas en Educación, 14(3), 1-24. Recuperado de http://revista.inie.ucr.ac.cr/index.php/aie/article/view/623

Monroy, Lucia, Tanamachi, María de Lourdes y Backhoff, Eduardo. (2005). Cuestionarios de Contexto para alumnos: Marco de Referencia, 2005. México. Instituto Nacional para la Evaluación de la Educación (INEE).

Muñoz Cantero, Jesús Miguel, Ríos de Deus, María Paula y Abalde, Eduardo. (2002). Evaluación docente versus Evaluación de la calidad. Revista ELectrónica de 
Investigación y EValuación Educativa, 8(2). Recuperado de http://www.uv.es/RELIEVE/v8n2/RELIEVEv8n2 4.htm

Murillo, Paulino. (2007). Nuevas formas de trabajar en la clase: metodologías activas y colaborativas. En Fernando Blanco (Dir.), El desarrollo de competencias docentes en la formación del profesorado (pp. 129-153). Madrid: MEC Colección Conocimiento Educativo.

Paredes, Joaquín. (1999). Análisis etnográficos de los usos de recursos de materiales didácticos en educación primaria: estudios de los casos de dos centros. (Tesis doctoral), Universidad Complutense de Madrid, Madrid, España.

Pozo, Juan Ignacio. (2008). Aprendices y maestros: la psicología cognitiva del aprendizaje. Madrid: Alianza Editorial.

Robles, María Isabel, Alfageme, María Begoña y Vallejo, Mónica. (2011). ¿Qué hacen los docentes en sus aulas?. El caso de un centro de educación primaria. Contextos educativos, 14, 49-65.

Sánchez, Primitivo. (2011). Métodos, principios y estrategias didácticas. En Isabel Cantón y Margarita Pino-Juste (Coords), Diseño y desarrollo curricular (pp. 185-204). Madrid: Alianza Editorial.

Solano, Luis. (2003). Informe de evaluación para potenciar el rendimiento académico en Educación Infantil y Primaria. INDIVISA.

Segura, Mario Alberto. (2009). La evaluación de los aprendizajes basada en el desempeño por competencias. Revista Actualidades Investigativas en Educación, 9(2), 1-25. Recuperado de http://revista.inie.ucr.ac.cr/index.php/aie/article/view/336

Tejada, José. (2011). ¿Qué son la didáctica y la teoría del currículum. En Isabel Cantón y Margarita Pino-Juste (Coords). Diseño y desarrollo curricular. (pp.19-40). Madrid: Alianza Editorial

Tourón, Javier. (2000). Evaluación de programas para alumnos de alta capacidad: algunos problemas metodológicos. Revista de Investigación Educativa, 18, 565-586. 


\section{Anexo 1}

Tabla 10

Escala Metodologías Docentes en Educación Primaria

Escala de Valoración: (1) Muy en desacuerdo al (4) Muy de acuerdo o desde (1) Nunca a (4) Siempre)

№ ITEM

DIMENSIÓN

ESCALA DE

VALORACIÓN

PLANIFICACIÓN PROCESO E/A

\begin{tabular}{l|l}
\hline 1 & El maestro o la maestra nos pide nuestra opinión para planificar lo que hacemo
\end{tabular} en clase.

2 Se nota que las clases son preparadas con antelación por el maestro o la maestra.

\begin{tabular}{|c|c|c|c|}
\hline TIPO ACTIVIDADES E/A & $\mathbf{1}$ & $\mathbf{2}$ & $\mathbf{4}$ \\
\hline
\end{tabular}

3 Las actividades que me gustan más son aquellas en las que puedo trabajar con otro compañero o compañera.

4 Las actividades que me gustan más son aquellas en las que hacemos debates.

5 Las actividades que me gustan más son aquellas en las que se exponen temas o trabajos en clase.

$6 \quad$ Las actividades que me gustan más son aquellas en las que realizamos trabajos de investigación y descubrimiento.

7 Las actividades que me gustan más son aquellas en las que salimos fuera del centro.

\begin{tabular}{l|l}
\hline 8 & El maestro o la maestra suele adaptar las actividades en función de nuestras
\end{tabular} necesidades e intereses.

\begin{tabular}{|c|c|c|c|c|c|}
\hline & ESTILO DOCENTE & 1 & 2 & 3 & 4 \\
\hline 9 & El maestro o la maestra presta ayuda adicional cuando la necesitamos. & & & & \\
\hline 10 & El maestro o la maestra responde con precisión a las preguntas que le hacemos. & & & & \\
\hline 11 & $\begin{array}{l}\text { El maestro o la maestra apoya el trabajo que realizamos (en clase, en casa o en } \\
\text { la biblioteca...). }\end{array}$ & & & & \\
\hline 12 & El maestro o la maestra ayuda al alumnado en su aprendizaje. & & & & \\
\hline 13 & $\begin{array}{l}\text { El maestro o la maestra continúa su explicación hasta que el alumnado lo } \\
\text { entienden. }\end{array}$ & & & & \\
\hline 14 & $\begin{array}{l}\text { El maestro o la maestra da al alumnado la oportunidad de expresar sus } \\
\text { opiniones. }\end{array}$ & & & & \\
\hline 15 & El maestro o la maestra nos motiva a participar en las actividades de clase. & & & & \\
\hline 16 & El maestro o la maestra nos premia cuando nos esforzamos en clase. & & & & \\
\hline 17 & $\begin{array}{l}\text { El maestro o la maestra nos premia cuando trabajamos en equipo y } \\
\text { colaboramos con nuestros compañeros o compañeras. }\end{array}$ & & & & \\
\hline 18 & $\begin{array}{l}\text { El maestro o la maestra nos premia cuando respetamos el trabajo de nuestros } \\
\text { compañeros/as. }\end{array}$ & & & & \\
\hline 19 & $\begin{array}{l}\text { El maestro o la maestra nos premia cuando atendemos durante las } \\
\text { explicaciones. }\end{array}$ & & & & \\
\hline 20 & $\begin{array}{l}\text { El maestro o la maestra nos premia cuando realizamos las tareas y ejercicios } \\
\text { para casa. }\end{array}$ & & & & \\
\hline 21 & El maestro o la maestra nos premia cuando cuidamos de los materiales. & & & & \\
\hline 22 & $\begin{array}{|lcccccc|}\text { El maestro } & 0 & \text { la maestra nos } & \text { premia } & \text { cuando } & \text { hacemos } \\
\text { presentaciones/exposiciones de trabajos. }\end{array}$ & & & & \\
\hline 23 & $\begin{array}{l}\text { El maestro o la maestra nos felicita o recompensa cuando actuamos } \\
\text { correctamente en clase. }\end{array}$ & & & & \\
\hline 24 & $\begin{array}{l}\text { El maestro o la maestra se enfada cuando no participamos o bloqueamos los } \\
\text { trabajos en equipo, interrumpiendo a los demás. }\end{array}$ & & & & \\
\hline 25 & $\begin{array}{l}\text { El maestro o la maestra se enfada cuando no respetamos el trabajo de los } \\
\text { compañeros o compañeras. }\end{array}$ & & & & \\
\hline 26 & $\begin{array}{l}\text { El maestro o la maestra se enfada cuando no atendemos durante las } \\
\text { explicaciones o nos distraemos. }\end{array}$ & & & & \\
\hline 27 & $\begin{array}{l}\text { El maestro o la maestra se enfada cuando no cuidamos los materiales propios o } \\
\text { de los compañeros o compañeras. }\end{array}$ & & & & \\
\hline 28 & $\begin{array}{llllllll}\text { El maestro } & \text { o la maestra se enfada cuando } & \text { no } & \text { realizamos } \\
\text { presentaciones/exposiciones adecuadas de los trabajos. } & & & \\
\end{array}$ & & & & \\
\hline 29 & maestro o & & & & \\
\hline
\end{tabular}


30 El maestro o la maestra nos castiga a través de comunicaciones a la familia.

31 Podemos preguntar todas las dudas en clase.

UTILIZACIÓN ESPACIOS Y TIEMPO

32 La distribución de mesas y sillas en clase favorece que todos nos comuniquemos fácilmente.

ESTRUCTURA Y ORGANIZACIÓN DE CONTENIDOS

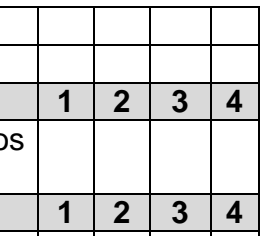

33 Normalmente cuando el maestro o la maestra comienza un tema nuevo suele exponer los objetivos que se pretenden alcanzar en el tema.

34 Normalmente cuando el maestro o la maestra comienza un tema nuevo suele repasar los conceptos de temas anteriores.

35 Normalmente cuando el maestro o la maestra comienza un tema nuevo suele explicar el tema de modo completo, para luego hacer ejercicios.

36 Normalmente cuando el maestro o la maestra comienza un tema nuevo suele buscar ejemplos de la vida diaria que faciliten la explicación del tema.

37 Normalmente cuando el maestro o la maestra comienza un tema nuevo suele entregar al alumnado material relacionado con el tema que se va a desarrollar.

\section{MATERIALES CURRICULARES Y OTROS RECURSOS}

38 Es habitual que en clase se utilicen apuntes de la pizarra o encerado.

39 Es habitual que en clase se utilicen libros de la biblioteca del colegio o del aula.

40 Es habitual que en clase se utilicen prensa escrita.

$\mathbf{4 1}$ Es habitual que en clase se utilicen los ordenadores.

42 Es habitual que en clase se utilicen medios audiovisuales (vídeo, proyector de diapositivas,...).

43 Es habitual que en clase se utilicen materiales elaborados por el propio maestro o la maestra (fichas, esquemas, apuntes...).

44 Es habitual que en clase se utilicen materiales elaborados en equipo (elaborados por el profesorado).

45 Es habitual que en clase se utilicen materiales impresos editados (libros de texto, mapas....).

46 Es habitual que en clase se utilicen material manipulativo (de laboratorio, juegos, instrumentos...).

\section{SENTIDO Y PAPEL EVALUACIÓN}

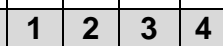

47 El maestro o la maestra nos informa de los criterios de evaluación que después aplica.

48 El maestro o la maestra nos evalúa a través de controles orales después de cada tema o lección.

49 El maestro o la maestra nos evalúa a través de controles escritos después de cada tema o lección.

50 El maestro o la maestra nos evalúa a través de controles en los que podemos utilizar material.

51 El maestro o la maestra nos evalúa a través de observarnos cuando trabajamos en clase.

52 El maestro o la maestra nos evalúa a través de los ejercicios que hemos hecho en el cuaderno.

53 El maestro o la maestra nos evalúa a través de la corrección de los deberes.

54 El maestro o la maestra nos evalúa a través de la nota que nosotros o nosotras mismas nos ponemos en los ejercicios y/o controles.

55 Nuestro maestro o maestra hace un seguimiento personal de cada estudiante.

56 La evaluación final es fruto del trabajo realizado a lo largo de todo el curso no sólo de los exámenes.

57 Nuestro maestro o la maestra explica la evaluación realizada revisándola si considera que puede haber un error.

58 Para sacar buenas calificaciones en mi clase es importante portarse bien

59 Para sacar buenas calificaciones en mi clase es importante hacer los deberes.

60 Para sacar buenas calificaciones en mi clase es importante hacer bien las actividades y trabajos.

61 Para sacar buenas calificaciones en mi clase es importante sacar buenas notas en los controles. 


\begin{tabular}{|l|l|c|c|c|}
\hline 62 & $\begin{array}{l}\text { Cuando un estudiante obtiene calificaciones muy bajas nuestro maestro o } \\
\text { maestra habla con él/ella. }\end{array}$ & & & \\
\hline $\mathbf{6 3}$ & $\begin{array}{l}\text { Cuando un estudiante obtiene calificaciones muy bajas nuestro maestro o } \\
\text { maestra suele darle tareas adicionales. }\end{array}$ & & \\
\hline $\mathbf{6 4}$ & $\begin{array}{l}\text { Cuando un estudiante obtiene calificaciones muy bajas nuestro maestro o } \\
\text { maestra suele avisar a los padres y madres. }\end{array}$ & & & \\
\hline \multicolumn{1}{|l|}{ VALORACIÓN GENERAL } & $\mathbf{1}$ & $\mathbf{2}$ & $\mathbf{3}$ & $\mathbf{4}$ \\
\hline $\mathbf{6 5}$ & $\begin{array}{l}\text { Creo que la forma de dar la clase del maestro o la maestra es adecuada para } \\
\text { que nosotros o nosotras aprendamos. }\end{array}$ & & \\
\hline
\end{tabular}

Fuente: Elaboración propia de las autoras (2014) 